\title{
Influence of synchronous and sequential stimulation on muscle fatigue
}

\author{
$\begin{array}{ll}\text { M. Thomsen } & \text { P. H. Veltink } \\ & \end{array}$ \\ ${ }^{1}$ Centre for Sensory-Motor Interaction (SMI). University of Aalborg, Fr. Bajersvej 7D-3, DK-9220 \\ Aalborg, Denmark \\ ${ }^{2}$ Department of Electrical Engineering, University of Twente, NL-7500 AE Enschede, The Nether- \\ lands
}

\begin{abstract}
-in acute experiments the sciatic nerve of the rat is electrically stimulated to induce fatigue in the medial Gastrocnemius muscle. Fatigue tests are carried out using intermittent stimulation of different compartments (sequential) or a single compartment (synchronous) of the sciatic nerve. The activation of different compartments is achieved by dividing nerve fibres into subbundles and placing them in separate grooves in a multigroove electrode. The aim of the investigation is to quantify the effect of sequential contra synchronised stimulation in reducing muscle fatigue, with no overlap between compartments. Overlap between two compartments is calculated using the combined and individual forces from both compartments. Sequential stimulation of two and three compartments is investigated. There is a significant decrease of fatigue in sequential stimulation compared to synchronous. After 2 min of intermittent stimulation the force time level is significantly increased in sequential stimulation, than in synchronous stimulation. The rate of force time decrease is significantly slower in sequential stimulation than in synchronous stimulation. With sequential stimulation it takes significantly longer before the maximal force time is reached than with synchronous stimulation.
\end{abstract}

Keywords-Electrical stimulation, Muscle fatigue, Overlap, Sequential, Synchronous

Med. Biol. Eng. Comput., 1997, 35, 186-192

\section{Introduction}

FUNCTIONAL ELECTRICAL stimulation (FES) is a technique that covers the application of electrical stimulation of the neuromuscular system for functional use, e.g. the restoration of mobility. One of the main problems in the area of FES is the rapid development of muscle fatigue during sustained or intermittent stimulation (PECKHAM et al., 1970; POURNEZAM et al., 1988; HAPPAK et al., 1989; YARKONY et al., 1992; FRANKEN et al., 1993). Fatigue may be defined as the inability of a muscle to continue the development or sustentation of a required force (TALONEN et al., 1990). Rapid muscle fatigue with electrical stimulation has several causes. Initially, the fast fatiguable motor units are recruited with electrical stimulation (PETROFSKY, 1978) in contrast with the physiological recruitment order. This can be overcome by the use of special stimulation techniques, anodal or high frequency blocking using tripolar cuff electrodes (BARATTA et al., 1989; FANG and MORTUMER, 1991; GOODALl et al., 1993). Secondly, stimulation pulses are normally given at a constant stimulation frequency, which may not be optimal to minimise fatigue for intermittent stimulation tasks. In bealthy subjects (BDDERMACLEOD and BARKER, 1991) and in cat experiments (BEVAN

First received 17 Juty 1995 and in final form 8 October 1996 Correspondence should be addressed to Morten Thomsen

O. IFMBE: 1997 et al., 1992) it appears to have the effect of letting the first few pulses in a burst be of high stimulation frequency. However, in patients with spinal cord injuries this effect appeared to be minor (FRANKEN et al., 1994). For both intermittent (FRANKEN et al., 1993) and continuous (GARLAND et al., 1988) stimulation it has been reported that when the lowest possible stimulation frequency is used, it will postpone fatigue. Thirdly, fast muscle fatigue occurs if a muscle is stimulated continuously (e.g. during stance). Boom et al. (BOOM et al., 1993) showed that intermittent stimulation, which gives on average the same contraction level as continuous stimulation, reduces fatigue.

A fourth important cause of excessive muscle fatigue during electrical stimulation is the synchronous activation of the motor units, whereas motor units in a normal, voluntary muscle contraction contract in a non-synchronised way. Several investigators (RACK and WESTBURY, 1969; PECKHAM et al., 1970; PETROFSKY, 1979; POURNEZAM et al., 1988; THOMA et al, 1989; TALONEN et al., 1990; YOSHIDA and HORCH, 1993a) have shifted the activation between different compartments of a muscle or a muscie group (sequential stimulation), trying to reproduce this behaviour of the central nervous system. They applied sequential stimulation in two ways: by stimulating one compartment for a certain period of time and thereafter switching to another compartment (POURNEZAM $e t$ al., 1988; THOMA et al., 1989), and by switching between compartments after each stimulation pulse (RACK and WESTBURY, 1969; PECKHAM et al., 1970; PETROFSKY, 1979; TALONEN et al., 1990; YOSHIDA and HORCH, 1993a). It has 
been shown that sequential stimulation minimises fatigue, when compared with synchronised stimulation (PECKHAM $e t$ al., 1970; POURNEZAM et al., 1988; THOMA et al., 1989; TALONEN et al., 1990; YOSHIDA and HORCH, 1993a). However, in these studies fatigue reduction was not related to the overlap degree of the compartments stimulated by different electrodes. In addition, a relation between the number of sequentially stimulated compartments and muscle fatigue has not been established.

The aim of this investigation is to quantify the effect of using sequential contra synchronised stimulation in reducing muscle fatigue, checking the overlap between compartments. The overlap is evaluated by stimulating one channel in the refractory period of another channel and comparing the resulting twitch force with the twitch forces of the individual channels (RUTTEN et al., 1991; YOSHIDA and HORCH, 1993b). We also investigate the influence of the number of sequentially stimulated compartments by comparing one, two and three compartments.

\section{Methods}

\subsection{Animal preparation}

In acute experiments we carry out the fatigue tests using 12 wistar rats (three months old or more). The animals are initially anaesthetised subcutaneously with Nembutal (70 $\mathrm{mg} \mathrm{kg}^{-1}$ body weight), subsequently we administer onequarter to one-fifth of the start-dose when needed. We control the depth of anaesthesia by checking the absence of comea reflexes and toe reflexes, and the frequency and depth of respiration. We expose the medial gastrocnemius muscle from the surrounding tissue and other muscles, leaving the blood supply, the innervating nerve and the proximal tendon intact. We cut the calcaneus bone, leaving a piece of bone attached to the Achilles tendon. We loop the tendon around a piece of suture attached to a force transducer (Harvard 383, range $0-10 \mathrm{~N}$ ); the muscle and tendon are then covered with paraffin oil to prevent drying.

We gently free the Sciatic nerve from connective tissue and split it in the farthest proximal direction. Then we place a multi-groove electrode configuration (Fig. 1) under the nerves, and with a fine pair of forceps (Dumont 5) and a pair of eye scissors (Teufel 4040-41) remove the outer epineurium from the Sciatic and the Tibial nerves. By a distal to proximal direction from the electrode configuration, we separate the nerve fibres belonging to the Peroneal and the Sural branches from the Sciatic nerve. The remaining fibres are divided into six subbundles and placed in separate grooves (channels). We perform this physical separation in bundles to assure maximal selectivity during these acute experiments. With a few test stimulations we find the maximal twitch force for each channel; a strongly responding channel is redivided, and weakly responding channels are pooled to give two or three subbundles of nerve fibres. To improve electrical separation, the grooves with the fbre bundles at the bottom are filled with Vaseline petroleum jelly, and we press silicone sheets into the filled grooves (Fig. 1).

To prevent reflex activation, we crush the Sciatic nerve 1-2 cm proximal to the electrode configuration.

Before starting the fatigue experiments, we test the loss of muscle twitch force by this procedure in four animals. We determine the maximal twitch force by stimulating the Sciatic nerve supra-maximally with a hook electrode. After the nerve bas been split, we determine the maximal twitch force by stimulating all channels simultaneously at a supra-maximal level.

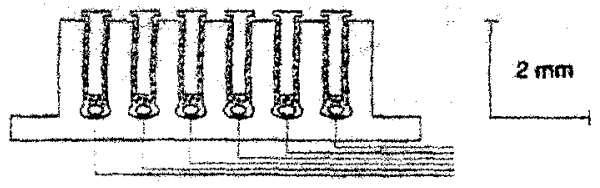

a

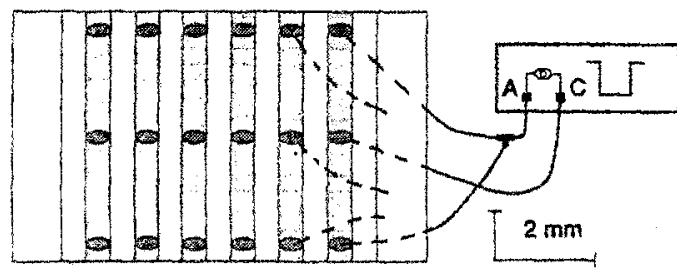

Fig. 1 Multi-groove electrode configuration; (a) side view; (b) top view; only the connection of three electrodes in one groove is shown: the electrodes in the other grooves are connected in the same way; $A$ indicates anode and $C$ indicates cathode

\subsection{Electrode configuration}

The electrode configuration is made of silicone rubber*and the electrode contacts of $90 \% \mathrm{Pt}$ and $10 \% \mathrm{Ir}$. The groove dimensions are: $10 \mathrm{~mm}$ long, $2 \mathrm{~mm}$ high and at the bottom approximately $0.5 \mathrm{~mm}$ wide. We place three electrodes at the bottom of each groove, one in the middle, and one in each end of the groove, $1 \mathrm{~mm}$ from the edge (Fig. 1). We give stimulation to the middle electrode (negative, monophasic, rectangular current pulses, pulse width $0.1 \mathrm{~ms}$ ) with reference to the short-circuited end electrodes, which are connected to a virtual ground (stimulators Hewlett Packard 3245A universal source)

\subsection{Experimental procedure}

During the experiments we place the animal on a heating table $\left(35^{\circ} \mathrm{C}\right)$, and control the temperature of the freed muscle and nerve by a warm airstream $\left(38-40^{\circ} \mathrm{C}\right.$ ) (WALLINGA-DE JONGE et al., 1980). The knee is rigidly clamped, the foot is pulled against the edge of the table, and the muscle is lifted so that it does not touch the surrounding tissue. At the beginning of the experiments we set the medial gastrocnemius muscle at optimal twitch length.

We perform recruitment curves on each channel. Depending on the difference between stimulation threshold and maximal response, monophasic stimulation pulses are increased in steps by a fixed value between 0.5 and $5(\mu \mathrm{A})$. For each responding channel we choose a stimulation current, which recruits a twitch force in the middle of a plateau on the recruitment curve. For the chosen stimulation currents we evaluate the overlap between neighbouring channels by activating twitch contractions (RUTTEN et al., 1991). We obtain a combined twitch force from two neighbouring channels by stimulating the two channels 400-1000 us apart with a stimulation of the second channel in the refractory period of the first channel. Each channel will maximally receive 160 twitch contractions delivered in blocks of 20 pulses at $1 \mathrm{~Hz}$. We give some test tetanic contractions before starting the fatigue tests. Each channel receives at least 40 tetanic contractions to ensure that fast fatigue and slow recovery fibres do not influence the fatigue tests.

\footnotetext{
- Dow Corning Silastic 734 FTV
} 


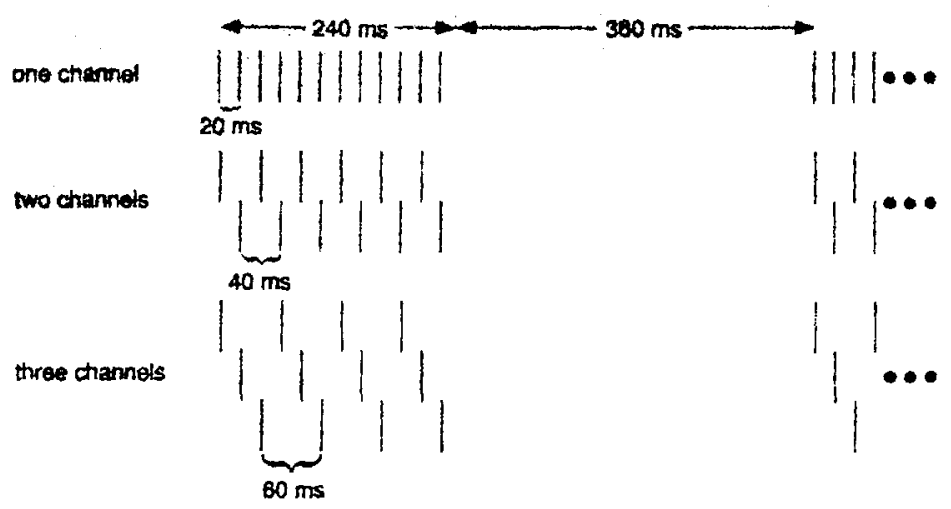

Fig. 2 Three different ways of performing fatigue trials; the stimulation regime is shown for one cycle

After a rest of at least 30 min we carry out fatigue tests. In between the fatigue tests we allow a recovery of at least $45 \mathrm{~min}$. The fatigue tests are carried out using intermittent stimulation. We adjust the intermittent fatigue test (BURKE et $a l, 1973)$ to the walking cycle of the rat using the following parameters: $0.6 \mathrm{~s}$ cycle time, $40 \%$ duty cycle, and $4 \mathrm{~min}$ fatigue test. We activate each tetanic contraction by 12 stimulation pulses, irrespective of the number of stimulation channels used; this gives an inter-pulse interval of $240 / 12 \mathrm{~m}$ (see Fig. 2), which corresponds to a stimulation frequency of $50 \mathrm{~Hz}$. In synchronous stimulation one channel is stimulated with $50 \mathrm{~Hz}$. In two channel sequential stimulation each channel receives six stimulation pulses of $25 \mathrm{~Hz}$ in each contraction. In three channel sequential stimulation each channel receives four stimulation pulses of $16.67 \mathrm{~Hz}$.

We carry out experiments by initially applying the threechannel sequential fatigue test (if it is possible to activate three channels selectively). Then we apply different combinations of two-channel sequential fatigue tests and finally singlechannel synchronous fatigue tests.

\subsection{Data analysis}

To evaluate the overlap between channels, we calculate a selectivity index (SI) by subtracting the twitch force of the channel with the highest twitch force $\left(F_{a}\right)$ from the combined twitch force $\left(F_{a b}\right)$, and dividing this by the twitch force from the channel with the lowest force $\left(F_{b}\right)$. Each of these three twitch forces has an average of $n=20$ stimulations:

$$
\mathrm{SI}=\frac{\frac{1}{n} \sum_{n} F_{a b}-\frac{1}{n} \sum_{n} F_{a}}{\frac{1}{n} \sum_{n} F_{b}}
$$

$\mathrm{SI}=1$ gives no overlap of stimulation, and $\mathrm{SI}<1$ indicates the overlap of stimulation. We evaluate the development of fatigue during a fatigue test using the average force (AF) of a tetanic contraction. We calculate a fatigue index as the quotient of AF for tetanic contraction number 200 (after $120 \mathrm{~s})$ and to the maximal $\mathrm{AF}\left(\mathrm{AF}_{\max }\right)$ :

$$
\mathrm{Fl}_{120}=\frac{\mathrm{AF} \mathrm{F}_{120}}{\mathrm{AF}}
$$

To estimate the rate of decreasing AF in a fatigue test, we find the maximally descending slope that occurs after $A F_{\max }$ has been found. We estimated the slopes by linear regression on 10 points.

The slope is calculated using a least-square technique to find the best linear intersection between 10 succeeding AFs.

We test statistical differences between groups of data at a $5 \%$ significant level using the non-parametric Mann-Whitney
$\mathrm{U}$ test. We use the Kendall rank-order correlation coefficient as a measure of correlation between groups of data.

\section{Results}

\subsection{Recruitment curves}

After the nerve has been divided into subbundles we test the loss of twitch force in four rats. The loss of twitch force never exceeds $10 \%$.

Plateaus at sub-maximal levels are observed in a majority of the recruitment curves; a typical result of this is shown in Fig. $3 a$. The chosen stimulation current for the fatigue tests is in the interval $250-400 \mu \mathrm{A}$. In a few recruitment curves this is not the case; the curve in Fig. $3 b$ is an example. In this case the stimulation current for the fatigue test is chosen to be $420 \mu \mathrm{A}$.

\subsection{Selectivity}

To test the selectivity between channels, we use two criteria to set the stimulation current: an amplitude that gives twitch force responses of comparable strength, and an amplitude that gives twitch force responses in the middle of a plateau. Fig. 4 shows a selectivity test between two neighbouring channels. In many experiments it is difficult to keep a constant output at a channel, as constant recruitment and derecruitment of fibres occur. Though some variation is found between the twitch amplitudes, they fluctuate around an average value. Table 1
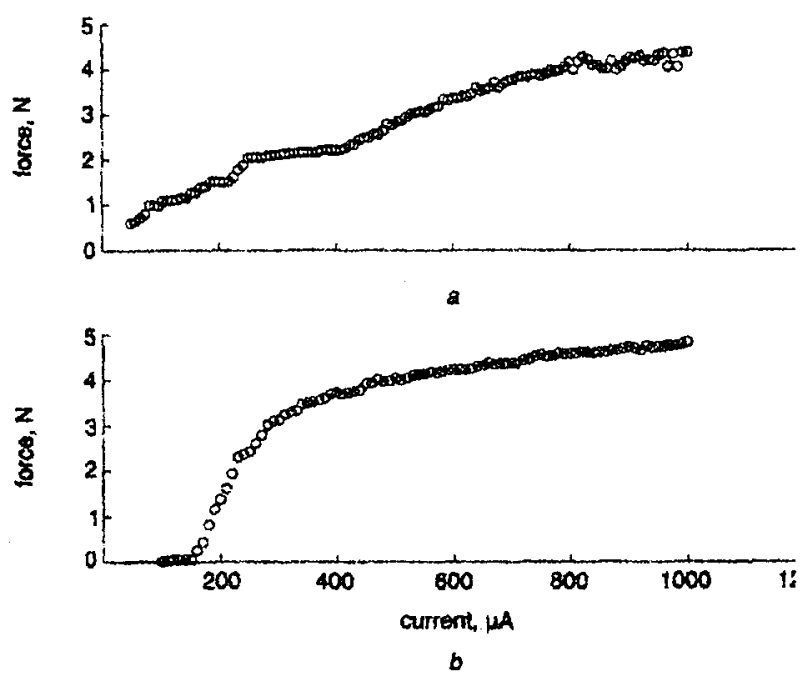

Fig. 3 Typical recruitment curves; (a) stimulation amplitude was increased in steps of $5 \mu \mathrm{A}$; (b) stimulation amplitude was increased in steps of $10 \mu \mathrm{A}$ 


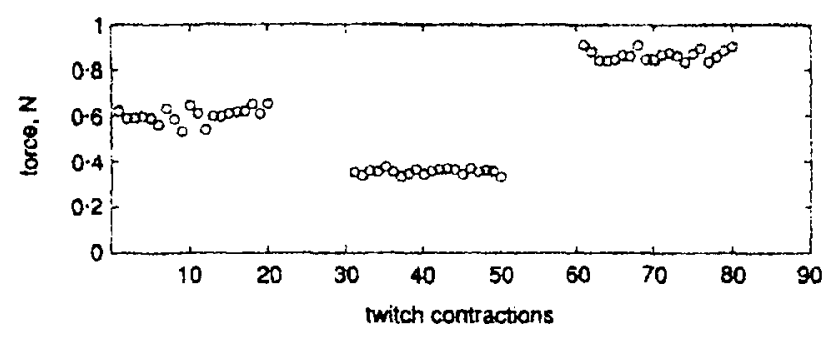

Fig. 4 Selectivity trials; the first 20 points are twitch forces from one channel, the next 20 points represent another channel, and the last 20 points are the combined stimulation of both channels stimulating the second channel in the refractory period of the first channel

gives selectivity parameters for channels participating in twochannel sequential fatigue tests. In two of the three-channel sequential fatigue tests, SI below 0.7 occurs between neighbouring channels (not shown in Table 1), and therefore a certain degree of overlap between channels takes place in this mode.

\subsection{Fatigue}

Fig. 5 depicts the fatigue tests with an animal in which we obtain three non-overlapping channels. At the beginning of the tests, potentiation of the AF takes place and an $\mathrm{AF}_{\max }$ is reached, after which the AF declines continuously. The onechannel tests fatigue more than the two-channel tests, and both two-channel tests fatigue more than the three-channel test. The potentiation has a longer duration in the three-channel test than in the one-channel tests; compared with the two-channel tests the difference is less pronounced (Fig. Sa). The difference between the first $A F$ and $A F_{\max }$ is smallest for the threechannel test (Fig. 5b). Synchronous stimulation gives smoother tetanic contraction than sequential stimulation. where mostly channels with different forces contribute to the tetanic contraction (Fig. 5b).

The latter part of some fatigue tests is rejected when a channel recruits new fibres during the test. If the new fibres are only recruited temporarily, the test is accepted. The recruitment of new fibres is determined by visually evaluating the tetanic contractions. During the $4 \mathrm{~min}$ intermittent fatigue, a major part of the channels changes recruitment, so we analyse tests only up to $2 \mathrm{~min}$.

We normalise the fatigue tests to their $A F_{\text {max }}$, and we pool all one-, two- and three-channel tests, as depicted in Fig. 6. The patterns of the fatigue development are the same as in Fig. 5.

Table 1 Selectivity parameters; only groups of stimulation channels used in the two channel fatigze tests are shown; in the second column the numbers of the two channels participating in the test are given; in the third column the force levels for each of the two channels are shown

\begin{tabular}{cccc}
\hline animal & channels & twitch forces, $N$ & selectivity \\
\hline 2 & 4 and 5 & $1.09-1.08$ & 0.98 \\
& 3 and 5 & $1.01-1.08$ & 0.98 \\
4 & 2 and 4 & $0.66-0.53$ & 0.93 \\
6 & 2 and 3 & $0.49-0.48$ & 0.97 \\
7 & 1 and 2 & $0.75-0.52$ & 0.90 \\
8 & 1 and 2 & $0.38-0.25$ & 0.93 \\
& 2 and 3 & $0.32-0.37$ & 0.93 \\
9 & 1 and 2 & $0.23-0.38$ & 1.01 \\
11 & 1 and 2 & $0.37-0.39$ & 0.89 \\
\hline
\end{tabular}

For animals with more than one active channel, $\mathrm{Fl}_{120}$ is always higher in sequential stimulation than in synchronous stimulation. In sequential stimulation, it takes more contractions before $A F_{m a s}$ is reached than in synchronous stimulation. In sequential stimulation, the rate of AF decrease is lower than in synchronous stimulation. When we compare the pooled fatigue parameters we find significant differences (Table 2).

The full and the broken lines in Fig. 7 show a least square fit of the dependence of $\mathrm{FI}_{120}(1$ channel $)$ and $\mathrm{FI}_{120}(2$ channel) on $\mathrm{Af}_{\max }$ respectively. The correlation coefficients are 0.16 for $\mathrm{FI}_{120}$ (1 channel) and 0.03 for $\mathrm{FI}_{120}(2$ channel). When independence is assumed, the one-tailed probabilities for correlations occurring higher than these is $21 \%$ and $46 \%$, respectively. Hence we find no evidence of a relationship between fatigue and force level.

To evaluate the influence of overlap between compartments in two channel fatigue tests, we plot $\mathrm{FI}_{120}(2$ channel) against the selectivity index (Fig. 8). In the range of SI (0.89-1.01), we find a slightly negative correlation $(-0.3)$ between the two parameters. Again with assumed independence, the one-tailed probability for a correlation occurring higher than this is $13 \%$. Hence, we find no evidence of a relationship between the degree of overlap observed in this investigation and twochannel sequential fatigue.

\section{Discussion}

In this study we compare sequential and synchronous fatigue tests. We obtain sequential stimulation by shifting stimulation between physically separated nerve-fibre bundles. Owing to a difference in twitch forces of the bundles an unfused tetanic contraction occurs in sequential stimulation (Fig. $5 b$ ). This could be a problem in some FES applications, such as the hand-grasp system (MEMBERG et al., 1993), but for a walking orthosis where large inertia needs to be moved the integral of the joint moments generated by the stimulated muscles is important (FRANKEN et al., 1995), and a minor ripple could be accepted. The procedure of dividing the nerve into subbundles would be impractical in an FES application, but if a synergistic muscle group needs to be activated, the innervating nerve branches of the different muscies can each be fitted with another type of stimulation electrode and activated in the same way as performed in this study.

After we have applied a fatigue test to a subbundle, a recovery period of $45 \mathrm{~min}$ is probably not enough for the subbundle to recover its full force output. As the aim of this investigation is to compare sequential to synchronous fatigue, full recovery is not critical so long as the subbundle responds to a fatigue test in the same way. CLAMANN and ROBNSON (CLAMANN and ROBINSON, 1985) reported that when the same fatigue test (BURKE et al., 1973) is applied several times to the same (cat) motor unit with intervals of $45 \mathrm{~min}$, the motor unit responded to the fatigue test in the same way.

When a subbundle is activated at a submaximal level, it can be difficult to keep a constant recruitment of motor units during a fatigue test. Out of 41 fatigue tests, 15 tests had to be rejected in calculating the fatigue index due to a change in recruitment within $2 \mathrm{~min}$. This is the reason why we choose such a short fatigue test in this investigation.

The relationship between stimulation frequency, the development of fatigue and the following recovery of cat motor units have been examined by CLAMANN and ROBINSON (CLAMANN and ROBINSON, 1985). They distinguish between low-frequency and high-frequency fatigue. In the literature the border between low- and high-frequency stimulation is rather floating, but the general rule seems to be that frequencies above $80 \mathrm{~Hz}$ in high-frequency stimulation are chosen. In two- 


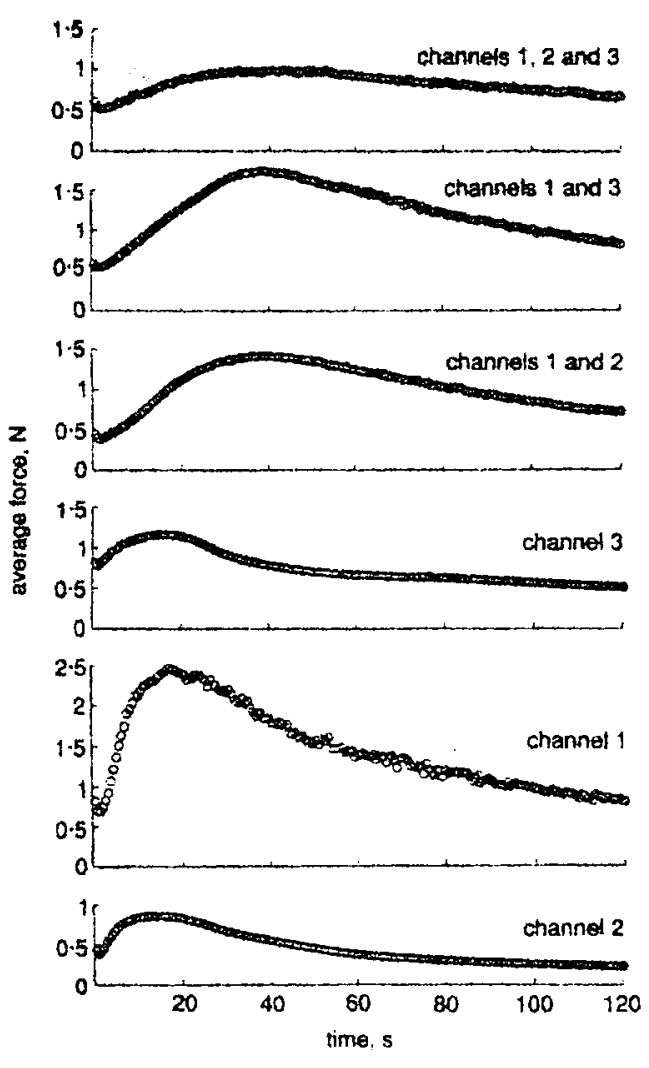

a
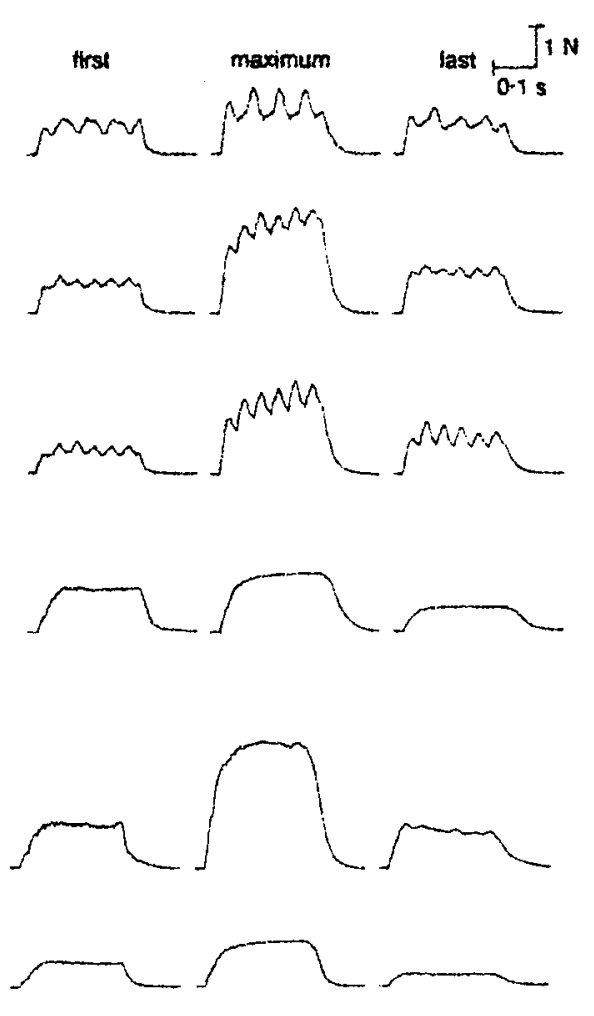

$b$

Fig. 5 Fatigue tests from one animal experiment (rat 8); the fatigue tests are dapicted in the order in which they were carried out with the top test being the first one and the bottom test being the last one; (a) the curves to the right represent average forces $(A F)$ of constituting tetanic contractions; (b) from left to right, the first tetanic, the maximal and the last contraction of the intermittent fatigue tests are show'n
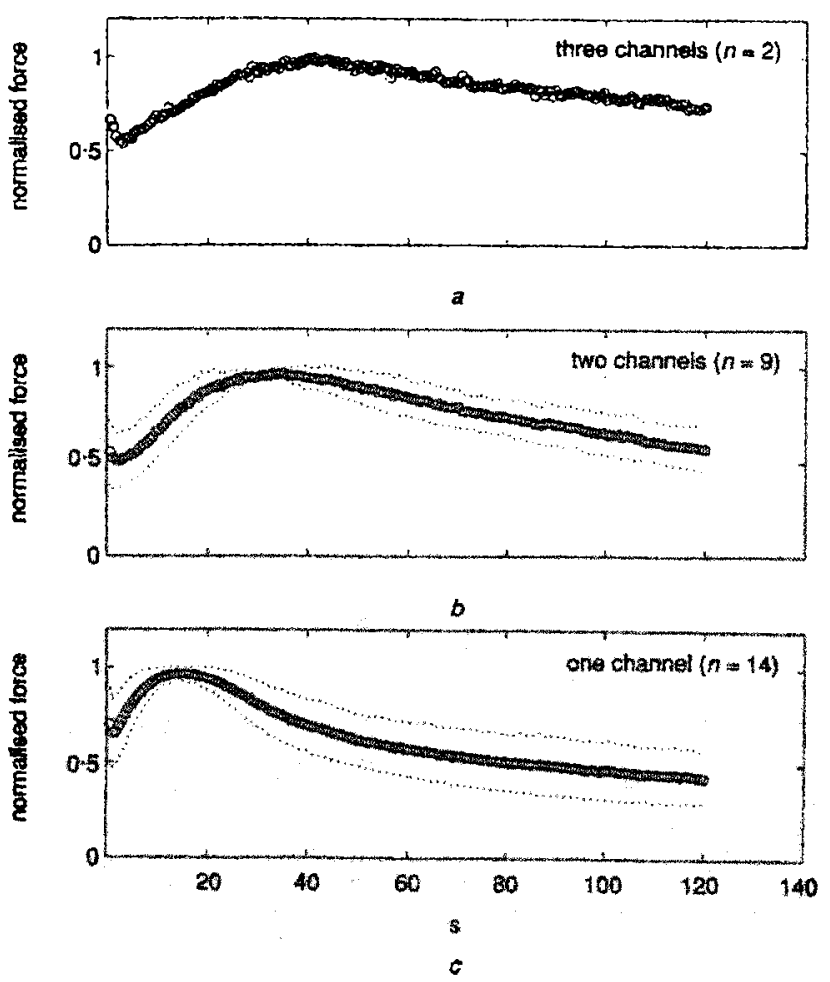

Fig. 6 Normalised fatigue tests; the circies represent the mean values of fatigue tests nomalised to their maximal, average force; the dots represent the standord deviation; the actual farigue parameters can be seen in Table 2 channel sequential mode each nerve compartment is stimulated by half the stimulation frequency used in synchronous mode. In three-channel sequential mode, each nerve compartment is stimulated by a third of the stimulation frequency used in synchronous mode. To develop the same kind of fatigue (low-frequency) in the synchronous and in the sequential modes, $50 \mathrm{~Hz}$ stimulation is used in synchronous mode.

The fatigue tests were carried out at different force levels. By electrical stimulation, the inverse recruitment order of motor units should lead to more fatigue and a fast decrease of force at a low recruitment level. According to Fig. 6, our results do not indicate any relation between force level and fatigue after 2 min. An explanation might be that in this experiment low force is not a result of low recruitment level, but of small bundle size.

YOSHIDA and HORCH (YOSHIDA and HORCH, 1993a) fatigued the gastrocnemius muscle in cats using Burke's fatigue test (BURKE et al., 1973) with a $60 \mathrm{~Hz}$ stimulation frequency. Their interleaved and single stimulation modes were analogous to the two-channel sequential and one-channel synchronous stimulation modes in this study. Though they reported overlap between the channels in interleaved stimulation (but not the degree of overlap), there is a good agreement with the results found in our investigation. Before a series of fatigue tests, they performed a conditioning of the muscle, which probably makes a minimal potentiation of the muscle, and this means that the initial force will be close to the maximal force. At the moment when single stimulation rectuces the initial force by $50 \%$, the interleaved stimulation reduces the initial force by approximately $25 \%$ (estimated from Fig. 2 in YOSHIDA and HORCH, 1993a). We find that two-channel sequential stimulation reduces $\mathrm{AF}_{\max }$ by $29 \%$ whereas synchronous stimulation reduces $\mathrm{AF}_{\text {max }}$ by $50 \%$ (Fig. 6). At this 
Table 2 Mean values for the considered farigue parameters; there is a significant improvement of fatigue parameters between synchronous and sequential modes; it is possible to observe an improvement in fatigue parameters in going from two to three channels, though this improvement is not significant in view of the small number of three-channel trials

\begin{tabular}{lccc}
\hline mode & $\mathrm{FI}_{120}$ & maximal slope $\times 10^{-2}$ & control for $\mathrm{AF}$ max $[\mathrm{s}]$ \\
\hline synchronous (one channel) & $0.43 \pm 0.14(15)$ & $1.26 \pm 0.37(17)$ & $17 \pm 6(17)$ \\
sequential (two channeis) & $0.57 \pm 0.11(9)^{*}$ & $0.59 \pm 0.18(10)^{*}$ & $35 \pm 8(11)^{*}$ \\
sequential (three channels) & $0.74 \pm 0.08(2)^{*}$ & $0.50 \pm 0.07(3)^{*}$ & $43 \pm 4(3)^{*}$ \\
\hline
\end{tabular}

* $5 \%$ significance between two- or three-channel sequential fatigue tests and one-channel synchronous fatigue tests.

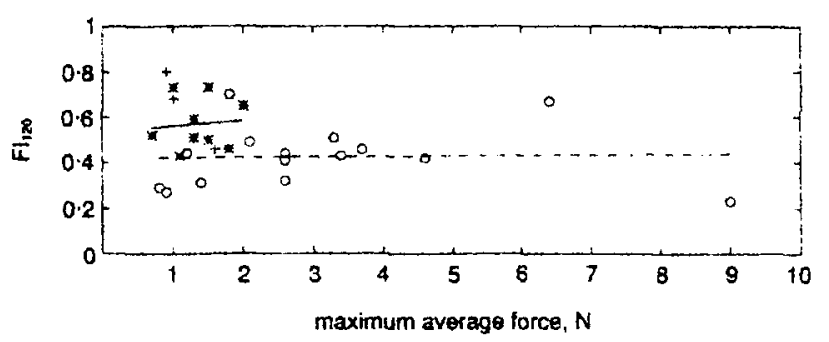

Fig. 7 Dependence of $\mathrm{Fl}_{120}$ on $A F_{\max }$; the symbols represent $A F_{\max }$ from the following fatigue tests; $O$ one-channel synchronous stimulation, * two-channel sequential stimulation and + three-channel sequential stimulation

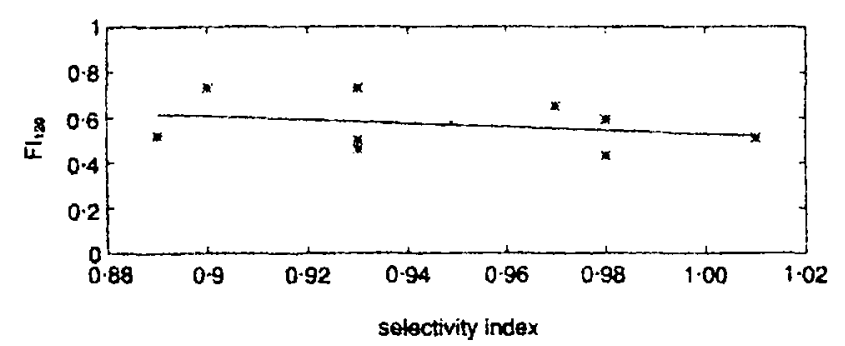

Fig. 8 Dependence of $F I_{I 20}$ (two channel) on selectivity index; the line shows a least square fit through the points

point three-channel sequential stimulation reduces $A F_{\text {max }}$ by $16 \%$. In the same investigation (YOSHIDA and HORCH, 1993a) the rate of fatigue between the 6th and 35 th contractions is calculated. It is reported that the rate of fatigue for interleaved stimulation is 0.42 (estimated from figure 3 of YOSHIDA and HORCH, 1993a) relative to single stimulation. We find that the maximal decreasing rate of fatigue for two-channel sequential stimulation is 0.47 , and for three-channel sequential stimulation it is 0.40 (Table 2) relative to synchronous stimulation.

THOMA and co-workers (HAPPAK et al., 1989; THOMA et al., 1989) fatigued sheep muscles using a modified version of Burke's fatigue test with a $40 \mathrm{~Hz}$ stimulation frequency. They did not examine the overlap between the channels in roundabout stimulation. Their round-about stimulation and single channel stimulation modes are comparable to the four-channel sequential and one-channel synchronous stimulation modes in this study. At the moment when single-channel stimulation reduces the initial force by $57 \%$, the round-about stimulation reduces the initial force by $34 \%$. The initial force was calculated as an average of the contractions in the first minute. From the example of the fatigue tests shown in Fig. 4 (THOMA et al., 1989) it seems that only a little potentiation occurred and the calculated average of the initial forces corresponds to $\mathrm{AF}_{\max }$. We find that when synchronous stimulation reduces $\mathrm{AF}_{\max }$ by $57 \%$, two-channel sequential stimulation reduces $\mathrm{AF}_{\text {maxx }}$ by $43 \%$ and three-channel sequential stimulation reduces $A F_{\max }$ by $26 \%$ (see Table 2 ).
The difference between the fatigue development of synchronous and sequential stimulation is significant. This applies for both the force level after 2 min of intermittent stimulation and the rate of force decrease. With sequential stimulation it takes the muscle significantly more tetanic contractions before it reaches maximal force than with synchronous stimulation.

If some fast-fatiguable slow-recovery fibres are active during the first fatigue trial and not in the following, this would not be in favour of the performance of three-channel fatigue, because it is always carried out first.

We cannot observe a significant difference in the fatigue parameters when going from two- to three-channel sequential stimulation. This is because of the low number of threechannel fatigue tests carried out, and also possibly the fact that in two out of three tests an overlap parameter of 0.6 is found between two neighbouring channels. We obtain three non-overlapping channels in only one animal (Fig. 4). In this animal the potentiation takes longer and the muscle fatigues less when comparing three-channel to two-channel sequential stimulation.

In this investigation we check the overlap between compartments. In contradiction to other studies we can conclude that the degree of overlap observed in this investigation does not influence the development of sequential fatigue (in the twochannel mode).

Acknowledgment-We wish to thank R. Busschers for his assistance and A. J. Verloop for the construction of the electrode. The research was performed during a stay of $M$. Thomsen at the University of Twente and was supported by the Danish Research Academy, the Danish National Research Foundation, the C. W. Obel fund, and the European programme Restoration of Muscle Activity through FES and Associated Technology (RAFT).

\section{References}

BARATTA, R., ICHIE, M., HWANG, S. K., and SOLOMONOV, M. (1989): 'Orderly stimulation of skeletal muscle motor units with tripolar nerve cuff electrode', IEEE Trans. Biomed. Eng., 36, (8), pp. 836843

BeVan, L., Laouris, Y., ReinKng, R. M., and Stuart, D. G. (1992): "The effect of the stimulation pattern on the fatigue of single motor units in adult cats', $J$. Physiol., 449, pp. 85-108

BINDER-MACLEOD, S. A, and BARKER, C. B. (1991): 'Use of a catchlike property of human skeletal muscle to reduce fatigue', Muscle Nerve, 14, pp. 850-857

BOOM, H. B. K., MULDER, A. J., and VelTINK, P. H. (1993): 'Fatigue during functional neuromuscular stimulation', Brain Res., 97, pp. $409-418$

BURKE, R. E., LEVINE, D. N., TSARIS, P., and ZaJaC, F. E. (1973): 'Physiological types and histochemical profiles on motor units of the cat gastrocnemius', J. Physiol, 234, pp. 723-748

Clamann, H. P., and Robinson, A. J. (1985): 'A comparison of electromyographic and mechanical fatigue properties in motor units of the cat hindlimb', Brain Res., 327, pp. 203-219

FANG, Z. P., and MORTMER, J. T. (1991): 'A method to effect 
physiological recruitment order in electrically activated muscle", IEEE Trans. Biomed. Eng., 38, pp. 175-179

FRANKEN, H. M., VELTINK, P. H., FIDUER, M., and BOOM. H. B. K. (1993): 'Fatigue of intermittently stimulated paralyzed human quadriceps during imposed cyclical lower leg movements', $J$. Electromyography Kinesiology. 3, (1). pp. 3-12

FRANKEN, H. M., VELTRK, P. H., GARN, VAN A., and BOOM, H. B. $K$. (1997) 'The influence of a stimulation pattern on force and fatigue profiles in FES-induced quadriceps contractions in paraplegics', J. Basic Applied Myology (in press)

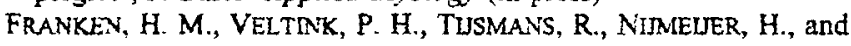
BOOM, H. B. K. (1995): 'Identification of quadriceps-shank dynamics using randomized interpulse interval stimulation', IEEE Truns., Rehub. Eng., 3, pp. 182-192

Garland, S. J., GARNER, S. H., and MCCOMAS, A. J. (1988): 'Relationship between numbers and frequencies of stimuli in human muscle fatigue', J. Appl. Physiol, 65, pp. 89-93

GOODALl, E. V., KOSTERMAN, L. M., STRUUT, J. J., and HOLSHEIMER, J. (1993): 'Simulation of activation and propagation delay? during tripolar neural stimulation'. Proc. 15th Ann. Int. Conf. IEEE/EMBS, San Diego, pp. 1203-1204

HAPPAK, W., GRUBER, H., HOLLE, J., MAYR, W., SCHUMUTTRERER, C. H., WINDBERGer, U., LOSERT, U., and THOMA, H. (1989): 'Multi-channel indirect stimulation reduces muscle fatigue'. Proc. Ith Ann. Int. Conf. IEEE Eng. in Med. Bisl. Soc., pp. 240-241

Memberg. W., PeCKham, P., Thrope, G., Keith, M., and KiCher, T. (1993): 'An analysis of the reliability of Percutaneous Electrodes in upper extremity FNS applications', IEEE Trans. Rehab Eng., 1, (2), pp. 126-132

PECKHAM, P. H., VAN DER MELLLeN, J. P., and ReswiCk, J. B. (1970): "Electrical activation of skeletal muscle by sequential stimulation' in WULFON, N., and SANCES, A (Eds.) The nervous system and electric currents (Plenum, New York), pp. 45-50

PETROFSKY, J. S. (1978): 'Control of the recruitment and firing frequencies of the motor units in electrically stimulated muscles in the cat', Med. Biol. Eng. Comput., 16, pp. 302-308

PETROFSKY, J. S. (1979): 'Sequential motor unit stimulation through peripheral motor nerves in the cat', Bed. Biol. Eng. Comput., 17, pp. $87-93$

Pournezam, M., Andrews, B. J., BAxendale, R. H., Phillips, G. F., and POUL, J. P. (1988): 'Reduction of muscle fatigue in man by cyclical stimulation', J. Biomed. Eny., 10, pp. 1988

RACK, P. M. H., and WESTBLRY, D. R. (1969): 'The effect of length and stimulus rate on tension in the isometric cat soleus muscle*, J. Physiol., 204, pp. 443-459

RUTtes, W. L. C., VAN Wier, H. J., and PUT, J. H. M. (1991): 'Sensitivity and selectivity of intraneural stimulation using a silicon electrode array', IEEE Trans. Biomed. Eng, 38, (2), pp. 192-198

TALONEN, P. P., Baer, G. A., HäkKNen, V., and OJala, J. K. (1990): 'Neurophysiological and technical considerations for the design of an implantable phrenic nerve stimulator', Med. Biol. Eng. Comput, 28. pp. 31-37

THOMA, H., GiRSCH, W., HOLLE, J., and MAYR, W. (1989): 'Technology and long-term application of an epineural electrode', Trans. Am. Soc. Artif. Intern. Organs, 35, pp. 490-494

Wallinga-de JoNGe, W., BOOM, H. B. K., Boon, K. L., GrieP, P. A. M., and LAMMEREE, G. C. (1980): 'Force development of fast and slow skeletal muscle at different muscle length', Am. $J$. Physiol, 239 (Cell Physiol. 8), pp. C98-C108

YARKONY, G. M., ROTH. E. J., CYBLLSKI, G., and JAEGER, R. J. (1992): 'Neuromuscular stimulation in spinal cord injury: I: Restoration of functional movement of the extremities', Arch. Phys. Rehabil, 73, pp. 78-86

YOSHDA, K., and HORCH, K. (1993a): 'Reduced fatigue in electrically stimulated muscle using dual channel intrafascicular electrodes with interleaved stimulation', Ann. Biomed. Eng., 21, pp. 709-714

YOSHIDA, K., and HORCH, K. (1993b): 'Selective stimulation of peripheral nerve fibers using dual intrafascicular electrodes', IEEE Trans. Biomed. Eng., 40, (5), pp. $492-494$

\section{Authors' biographies}

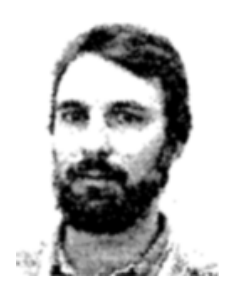

Morten Thomsen received an MSc degree in electrical engineering from Aalborg University, Aalborg, Denmark, in 1991 (topic: muscle spasticity in Hemiplegic patients). From 1991 to 1992 he investigated muscie fatigue at University of Twente, the Netherlands. From 1993 to 1997 he worked at Aalborg University to obtain a PhD. His research is currently in the area of analysing and optimising cuff electrodes for recording of nerve signals (used as a sensor in functional neuromuscular stimulation).

Peter $H$. Veltink received an MSc degree in electrical engineering from the University of Twente, Enschede, the Netherlands, in 1984 and $\mathrm{a} \mathrm{PhD}$ in 1988 (topic: recruitment of myelinated nerve fibres during artificial nerve stimulation). Currently, he is a faculty member of the Biomedical Engineering Department of the Faculty of Electrical Engineering, University of Twente. His research is in the area of control of functional aeuromuscular stimulation and the application of body mounted sensors for human movement analysis. 